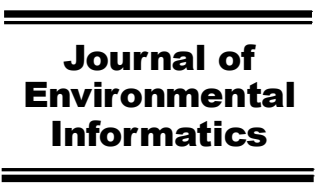

www.iseis.org/jei

\title{
MCFP: A Monte Carlo Simulation-based Fuzzy Programming Approach for Optimization under Dual Uncertainties of Possibility and Continuous Probability
}

\author{
B. Chen ${ }^{1,2, ~ *, ~ P . ~} \mathrm{Li}^{2}$, H. J. Wu${ }^{2}$, T. Husain ${ }^{2}$, and F. Khan ${ }^{2}$ \\ ${ }^{I}$ Key Laboratory of Regional Energy and Environmental Systems Optimization, Ministry of Education, Resources and Environmental Research \\ Academy, North China Electric Power University, Beijing 102206, China \\ ${ }^{2}$ Faculty of Engineering and Applied Science, Memorial University of Newfoundland, St. John's NL A1B 3X5, Canada
}

Received 30 July 2013; revised 8 January 2014; accepted 1 August 2014; published online 1 March 2015

\begin{abstract}
The efficiency and confidence of decision making much rely on accurate information and objective judgement, however, which are usually compromised by uncertainties existing in the system. Although in many studies uncertainties are reflected during optimization processes, few models considered the dual uncertainties of possibility and continuous probability. This study proposed a Monte Carlo simulation-based fuzzy programming (MCFP) approach to handle such dual uncertainties. The developed approach was tested by a municipal solid waste management (MSW) problem to demonstrate its feasibility and efficiency. The results indicated that the proposed approach could obtain a reliable solution and adequately support the decision making process in MSW management. It is significantly advantageous in handling the coexistence of various fuzzy sets and complex probability distributions when compared to the conventional fuzzy stochastic programming approaches. Furthermore, three levels of the optimal results to help decision makers effectively manage the composting facility: the entire distributions for general policy makers in long term policy making and trade-off, risk and reliability analyses of the system; the range of most frequent occurrences for project/plant managers in a medium arrangement; and the expected values for the plant operators for short term operating and adjusting the facility to minimize the system cost. Such different levels of decision supports could make the MCFP approach highly feasible, flexible and adaptable in real-work applications.
\end{abstract}

Keywords: dual uncertainties, possibility, probability, Monte Carlo simulation, optimization, municipal waste management (MSW)

\section{Introduction}

In environmental management, the efficiency and confidence of decision making much rely on accurate information and objective judgement, however, which are usually compromised by uncertainties existing in the systems (Cheng et al., 2009; Li and Chen, 2011; Jing et al., 2013; Tan et al., 2013). Such uncertainties may arise from a variety of possible sources in a management system including incomplete information, measurement and sampling errors, subjective judgement, assumptions and approximation, dynamics of environmental conditions etc. (Huang, 1998; Chen et al., 2008; Ping et al., 2010a; Li et al., 2011). Traditional deterministic programming methods may lack power to efficiently support decision making due to their weakness in reflecting the above uncertainties ( $\mathrm{Li}$ and Chen, 2011; Li et al., 2014). The growing interests and needs in how to reflect and quantify uncer tainties from different sources in the envir onmenttal management system have arisen in recent years (Li et al., 2012).

* Corresponding author. Tel.: +1 709 8648958; fax: +1 7098644042.

E-mail address: bchen@mun.ca (B. Chen).

ISSN: 1726-2135 print/1684-8799 online

(c) 2017 ISEIS All rights reserved. doi:10.3808/jei.201500293
Usually, uncertainties can be classified into two categories: possibilistic and probabilistic, which are commonly represented by the fuzzy set theory and stochastic system, respectively (Ramik and Vlach, 2004; Lin et al., 2009; Liu et al., 2009). Fuzzy techniques can be used to express the possibilistic type of uncertainties where vagueness of parameters is characterized by membership functions (Qin and Huang, 2008; $\mathrm{Xu}$ et al., 2009; Yang et al., 2010). Stochastic techniques can handle the probabilistic uncertainty in which the probability distributions are used to represent random variability of parameters (Blair et al., 2001; Seuntjens, 2002; Baudrit et al., 200 7). However, membership functions might lead to loss of information when some parameters could be represented by stochastic variables and/or when inappropriate subjective judgement was involved; and the definition of probability distributions could suffer from lack of sufficient data (Li et al., 2007; Qin and Huang, 2008; Yang et al., 2010). Furthermore, the two types of uncertainties frequently coexist (named dual uncertainties) in environmental systems, such as municipal solid waste (MSW) management system. Consequently, the integration of both methods has been considered in literature (Cheng et al., 2009). However, the previous studies usually faced difficulties in linking these two algorithms and appropriately interpreting of the relevant results (e.g., fuzzification and defuzzification are complex when the stochastic methods 
are involved). Therefore, many of these studies treated dual uncertainties separately instead of integratively (Liu et al., 2004; Li et al., 2006; Qin and Huang, 2008; Yang et al., 2010).

There were some attempts to deal with possibilistic and probabilistic uncertainties simultaneously. For instance, Huang et al. (2001) proposed an integrated fuzzy-stochastic linear programming model which could effectively deal with different types of uncertainties in optimization process and could obtain reasonable and reliable solutions under different significant levels. Guo and Huang (2009) proposed an approach to consider the dual uncertainties in water resource management by describing the parameters as probability distributions and fuzzy sets. They also proposed a concept of distribution with fuzzy probability (DFP) to reflect the dual-uncertainty characteristics of parameters. Li et al. (2009) proposed an inexact fuzzy-stochastic constraint-softened programming method to deal with possibilistic and probabilistic uncertainties, and applied to long term planning of a MSW management system. Based on a multistage fuzzy-stochastic integer programming model, a fuzzy-stochastic-based violation analysis approach was developed by Li and Huang (2009) to help water resources management.

These studies proposed some possible solutions to handle dual uncertainties of possibility and probability. However, they were significantly restrained on how to simultaneously deal with subjective information and continuous stochastic variables (presented by fuzzy sets and probability density functions, respectively) (Yang et al., 2010). In order to address the limitation in treating continuous stochastic variables, Monte Carlo simulation can be used to generate sufficient inputs to solve the insufficient data problems if the probability density function (PDF) could be accurately estimated or subjectively selected (Freeze et al., 1991; Vose, 1996; Garthwaite et al., 2005; Karmperis et al., 2012). In real-world situations, the continuous stochastic variables usually include subjective and objective information, leading to the dual uncertainties of possibility and continuous probability. To handle such dual uncertainties is beyond the ability of Monte Carlo simulation itself $(\mathrm{Gu}-$ yonnet et al., 2003; Goldstein, 2006; Yang et al., 2010; Li et al., 2011; Li et al., 2012). The integration of fuzzy programming with Monte Carlo simulation could be a promising solution (Sadeghi et al., 2010; Li et al., 2013). However, due to the difficulties in integrating fuzzy programming with Monte Carlo simulation, only a few studies were reported and they were all used to assess health risk issues (Guyonnet et al., 2003; Li et al., 2004; Chen et al., 2003; Liu et al., 2004; Li et al., 2007; Sadeghi et al., 2010; Ping et al., 2010b). In addition, because of the complex iterations in optimization algorithm, the integration of fuzzy programming and Monte Carlo simulation becomes challenging, and none such study is applied in optimization.

The objective of this study is to develop a Monte Carlo simulation-based fuzzy programming (MCFP) approach and the associated solution algorithm, effectively reflecting the dual uncertainties of possibility and probability in management systems.

\section{Methodology}

A fuzzy-stochastic-interval linear programming (FSILP) method was previously introduced by the authors ( $\mathrm{Li}$ and Chen, 2011). It was an advance from the Van Hop's approach intervals (Van Hop, 2007a, b, c), which was developed aiming at handling the coexistence of the uncertainties in forms of fuzzy set, and random values. One significant advantage of the FSILP method is that it can effectively present uncertainties in terms of fuzzy membership functions and probability density functions to incorporate both uncertain information and subjective judgement into a general framework. It has advantageous capabilities in easily achieving the optimal solutions with fewer additional constraints, leading to significant reduction of computation time and of complexity in solution. The following shows the integration algorithm of the FSILP method (Li and Chen, 2011):

Consider a fuzzy stochastic linear program as follows:

Min $f=C X$

Subject to:

$\sum_{j=1}^{n}\left(\tilde{A_{i j}}\right)_{w} X_{j} \leq\left(\tilde{B_{i}}\right)_{w}, \quad i=1, \cdots, m$

$X_{j} \geq 0, w \in \Omega$

where $C \in\{R\}^{1 \times n}$ is the matrix of coefficients of the objective function; $X$ is the matrix of decision variables; $A_{i j} \in\{R\}^{m \times n}$ is the matrix of fuzzy random variable constraint coefficients; $B_{i} \in\{R\}^{m \times 1}$ is the matrix of fuzzy random resources in constraints; $w$ denotes the probabilistic uncertainty defined on a probability space $(\Omega, F, P) ; n$ is the number of decision variables; and $m$ is the number of constraints. Assuming all fuzzy numbers are in the form of $t=\left(t, \delta^{-}, \delta^{+}\right)$, where $t$ is the most likely value, and $\delta^{-}$and $\delta^{+}$are the lower and upper spreads of the membership function, according to Van Hop (2007c), Equation (1) can be converted to:

Min $f=C X+E\left[\sum_{i=1}^{m} \lambda_{i}^{1}(w)\right]-E\left[\sum_{i=1}^{m} \lambda_{k}^{2}(w)\right]$

Subject to:

$$
\begin{aligned}
& \left.\frac{1}{2}\left(\sum_{j=1}^{n}\left(\tilde{A_{i j}^{1}}\right)_{w} X_{j}+\delta X_{j}\right)+\delta-\left(\tilde{B_{i}^{1}}\right)_{w}\right)=\lambda_{i}^{1}, \quad i=1, \cdots, u \\
& \frac{1}{2}\left(\sum_{j=1}^{n}\left(\left(\tilde{A_{k j}^{2}}\right)_{w} X_{j}-\delta X_{j}\right)-\delta-\left(\tilde{B_{k}^{2}}\right)_{w}\right)=\lambda_{k}^{2}, \quad k=1, \cdots, v \\
& X_{j}, \delta, \lambda_{i}^{1}, \lambda_{k}^{2} \geq 0, w \in \Omega
\end{aligned}
$$


where $\lambda_{i}^{1} \in\{R\}^{u \times 1}$ and $\lambda_{k}^{2} \in\{R\}^{v \times 1}$ are matrices of control decision variables corresponding to the degree (membership grade) to which $X$ solution fulfils the fuzzy constraints; and $E$ denotes the mathematical expectation; $A_{i j}^{1} \in\{A\}^{u \times n}$ and $A_{k j}^{2} \in$ $\{A\}^{v \times n}$ are matrices of positive and negative coefficients in the constraint, respectively; $B_{i}^{1} \in\{R\}^{u \times 1}$ and $B_{k}^{2} \in\{R\}^{v \times 1}$ are matrices of positive and negative right-hand-sides (RHSs); $u$ is the number of constraints with positive coefficients and RHSs; and $v$ is the number of constraints with negative coefficients and RHSs.

The Equation (2) is then derandomized by using stochastic programming techniques. The corresponding deterministic model for this problem is:

$\operatorname{Min} f=C X+\sum_{i=1}^{m} p_{i}^{1} \lambda_{i}^{1}-\sum_{i=1}^{m} p_{k}^{2} \lambda_{k}^{2}$

Subject to:

$\frac{1}{2}\left(\sum_{j=1}^{n}\left(\left(\tilde{A_{i j}^{1}}\right)_{w} X_{j}+\delta X_{j}\right)+\delta-\left(\tilde{B_{i}^{1}}\right)_{w}\right)=\lambda_{i}^{1}, \quad i=1, \cdots, u$

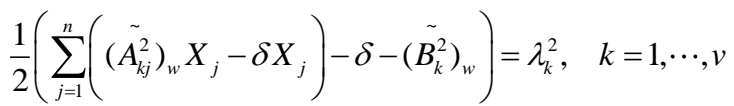

$X_{j}, \delta, \lambda_{i}^{1}, \lambda_{k}^{2}, p_{i}^{1}, p_{k}^{2} \geq 0, w \in \Omega$

where $p_{i}^{1} \in\{R\}^{m \times 1}$ and $p_{k}^{2} \in\{R\}^{o \times 1}$ are matrices of probabilities for random variables.

The Van Hop's method only considered the situation when the demands (left-hand-sides, LHSs) and sources (RHSs) were close, with LHSs $\leq$ RHSs in minimization problems or LHSs $\geq$ RHSs in maximization problems. In the situation that sources/RHSs are too abundant to be met by the demands/LHSs, the conversions from less-than signs to equal signs would lead to problematic and possible errors by Van Hop's method. In order to fix this problem, the slack variable is added in the loosing constrains as follows ( $\mathrm{Li}$ and Chen, 2011):

$\operatorname{Min} f=C X+\sum_{i=1}^{m} p_{i}^{1} \lambda_{i}^{1}-\sum_{i=1}^{m} p_{k}^{2} \lambda_{k}^{2}$

Subject to:

$$
\begin{aligned}
& \left.\frac{1}{2}\left(\sum_{j=1}^{n}\left(\left(\tilde{A_{i j}^{1}}\right)_{w} X_{j}+\delta X_{j}\right)+\delta+S_{i}-\tilde{B_{i}^{1}}\right)_{w}\right)=\lambda_{i}^{1}, \quad i=1, \cdots, u \\
& \frac{1}{2}\left(\sum_{j=1}^{n}\left(\left(\tilde{A_{k j}^{2}}\right)_{w} X_{j}-\delta X_{j}\right)-\delta-\left(\tilde{B_{k}^{2}}\right)_{w}\right)=\lambda_{k}^{2}, \quad k=1, \cdots, v \\
& \lambda_{i}^{1}, \lambda_{k}^{2} \leq \frac{1}{2}\left(\sum_{j=1}^{n} \delta X_{j}+\delta\right)
\end{aligned}
$$

$X_{j}, \delta, \lambda_{i}^{1}, \lambda_{k}^{2}, p_{i}^{1}, p_{k}^{2}, S_{i} \geq 0, w \in \Omega$

where $S_{i} \in\{R\}^{m \times 1}$ is the matrix of the slack variables. The constraints of $\lambda_{i}^{1}, \lambda_{k}^{2} \leq 1 / 2\left({ }^{n} \sum_{j=1} \delta X_{j}+\delta\right)$ are added because $\lambda$ represents the attainment of the memberships of LHS and RHS which is also equivalent to the overlap of these two memberships on one side spread.

Although the FSILP method is capable of handling the coexistence of dual uncertainties, its efficiency will decrease when the number of discrete probabilities increases. Furthermore, when the uncertainty is described as continuous probability, integration is required when numerically processing the optimization, leading to difficulties. Furthermore, some of the distributions may be non-integrable, making the optimization unachievable.

Monte Carlo methods are a class of computation intensive algorithms based on randomization. These methods can provide equivalent results to deterministic algorithms, which makes it a complement to the theoretical derivations (Anderson, 1986). Monte Carlo methods are especially suitable for the problems with multiple probability distributions, and the handling of such distributions becomes complicated by using numerical methods. These methods are frequently used to treat uncertainties in inputs, especially for evaluating risks (Baeurle, 2009).

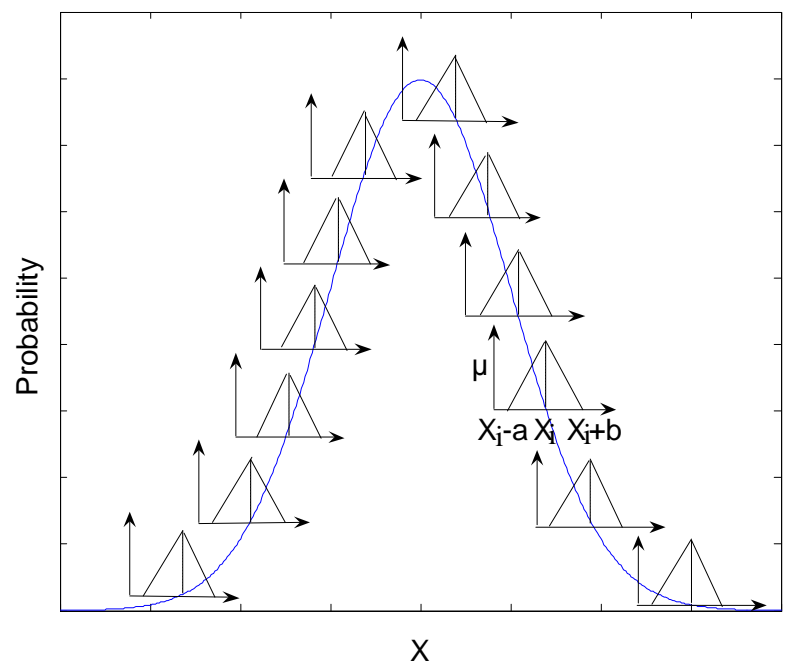

Figure 1. Dual uncertainties of possibility and contiuous probability.

The results of an objective function can be regarded as a stochastic one due to randomness of the input parameters. The occurrence of this can be predicted through Monte Carlo simulation-based on the help of the probability concept. However, not all the input parameters can be characterized by using probability distributions due to incomplete or insufficient information from literature and historical data as well as the subjective judgement when choosing values for the parameters. In many cases, the obtained probability distribution may be still uncertain where each data point contains a degree of belief, leading 


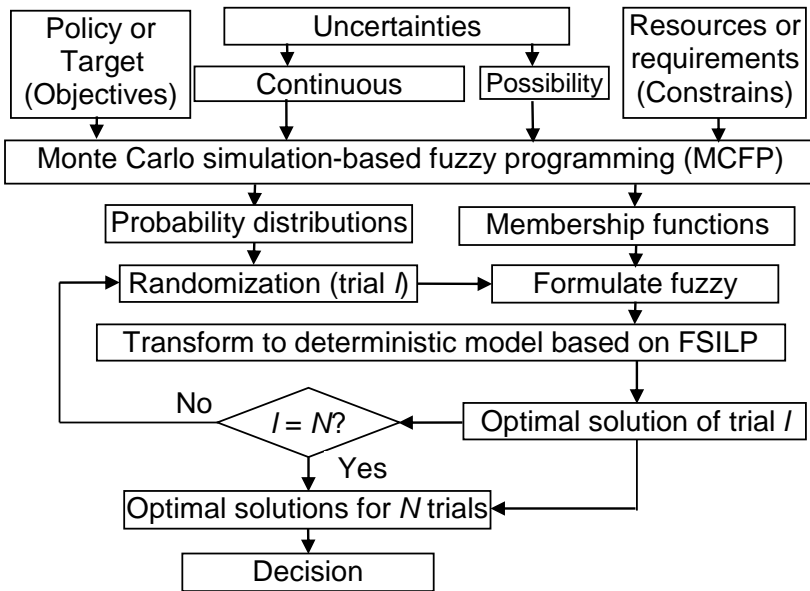

Figure 2. Framework of the Monte Carlo simulation-based fuzzy programming (MCFP).

to dual uncertainties of possibility and continuous probability.

As shown in Figure 1, a parameter $X$ is uncertain with corresponding probability:

$X \in \mathfrak{R} \mapsto X=f^{-1}(P)$

However, sometimes the confidence of such a distribution can be impaired by insufficient information. Such a consequence is of a fuzzy nature which can be quantified by degrees of belief (e.g., membership functions) (Li et al., 2007). Each data point $\left(y_{i}\right)$ may contain a membership function as follows:

$$
y_{i}=\left\{\tilde{t} ; \tilde{t}=\left(t, \delta^{-}, \delta^{+}\right), \delta^{-}, \delta^{+} \geq 0\right\}
$$

and

$$
\mu_{t}\left(y_{i}\right)= \begin{cases}\max \left(0,1-\frac{t-y}{\delta^{-}}\right), & \text {if } y \leq t \\ 1, & \text { if } \delta^{-}=0, \delta^{+}=0, t=y \\ \max \left(0,1-\frac{y-t}{\delta^{+}}\right), & \text {if } y \leq t \\ 0, & \text { otherwise }\end{cases}
$$

Therefore, in order to effectively tackle such coexistence of dual uncertainties, Monte Carlo simulation and fuzzy programming need to be integrated. The FSILP method can easily convert a fuzzy problem into a deterministic one without traditional fuzzification and defuzzification processes which significantly obstructs the integration with Monte Carlo simulation. The framework of the Monte Carlo simulation-based fuzzy programming (MCFP) is shown in Figure 2, where $N$ is the preset number of trials, and $l$ is the index of the current trial.

Consider a problem which is the same as the one in Equation (1). The random values of the parameters are firstly assi- gned in each Monte Carlo simulation trial according to their probability distributions, leading only to a fuzzy problem in each trial. According to the FSILP approach, in each trial the problem can be converted as follows ( $\mathrm{Li}$ and $\mathrm{Chen}, 2011$ ):

$\operatorname{Min} f=C X+\sum_{i=1}^{m} \lambda_{i}^{1}-\sum_{i=1}^{m} \lambda_{k}^{2}$

Subject to:

$$
\begin{aligned}
& \frac{1}{2}\left(\sum_{j=1}^{n}\left(\tilde{A}_{i j}^{1} X_{j}+\delta X_{j}+\delta\right)+S_{i}-\tilde{B_{i}^{1}}\right)=\lambda_{i}^{1}, \quad i=1, \cdots, u \\
& \frac{1}{2}\left(\sum_{j=1}^{n}\left(\tilde{A_{k j}^{2}} X_{j}-\delta X_{j}-\delta\right)-\tilde{B_{k}^{2}}\right)=\lambda_{k}^{2}, \quad k=1, \cdots, v \\
& \lambda_{i}^{1} \leq \frac{1}{2}\left(\sum_{j=1}^{n} \delta_{x_{j}}^{+} X_{j}+\delta_{B_{i}^{1}}^{-}\right) \\
& \lambda_{k}^{2} \leq \frac{1}{2}\left(\sum_{j=1}^{n} \delta_{x_{j}}^{-} X_{j}+\delta_{B_{k}^{2}}^{+}\right) \\
& X_{j}, \delta, \lambda_{i}^{1}, \lambda_{k}^{2}, p_{i}^{1}, p_{k}^{2}, S_{i} \geq 0, w \in \Omega
\end{aligned}
$$

After $N$ trials are finished, the sets of the results can be obtained as follows:

$$
f_{l, o p t}=\left\{f\left(X_{j l, o p t}\right) ; X_{j l, \text { opt }} \geq 0\right\}, \quad l=1, \cdots, M ; j=1, \cdots, Z
$$

where $M$ is the number of the feasible solutions after $N$ trials of the Monte Carlo simulation, and $Z$ is the number of decision variables.

Assuming that there is no uncertainty existing in the coefficients of the objective function $(C)$, the definition for the final solution can be stated as follows:

\section{Definition 1:}

$$
E\left(f_{l, o p t}\right)=\left\{f\left(E\left(X_{j, o p t}\right)\right) ; E\left(X_{j, o p t}\right) \geq 0\right\}, \quad l=1, \cdots, M
$$

Proof. the corresponding objective function and decision variables are:

$$
\begin{aligned}
& f_{l, o p t}=\left\{f\left(X_{j l, o p t}\right) ; X_{j l, o p t} \geq 0\right\}=\sum_{j=1}^{Z} C_{j} X_{j l, o p t}, \quad l=1, \cdots, M \\
& \Leftrightarrow E\left(f_{l, o p t}\right)=\sum_{j=1}^{Z} E\left(C_{j} X_{j l, o p t}\right)
\end{aligned}
$$

Since $C_{j}$ are deterministic and independent, we have the relation between the expected results of the optimal function and the decision variables:

$E\left(f_{l, o p t}\right)=\sum_{j=1}^{Z} C_{j} E\left(X_{j l, o p t}\right)=f\left(E\left(X_{j l, o p t}\right)\right)$ 
Table 1. Inputs for the Case of Decision Support to MSW Management

\begin{tabular}{lllll}
\hline Para. & Unit & $\begin{array}{l}\text { Deterministic } \\
\text { /expect value }\end{array}$ & $\begin{array}{l}\text { Normal } \\
\text { Distribution }\end{array}$ & Membership \\
\hline $\mathrm{c}_{1}$ & $\$ 10^{3} /$ tonne & 3 & $/$ & $/$ \\
$\mathrm{c}_{2}$ & $\$ 10^{3} /$ tonne & 2 & $/$ & $/$ \\
$\mathrm{a}_{11}$ & $\begin{array}{l}\text { ton of } \\
\text { waste/tonne }\end{array}$ & 3 & $/$ & $(\mathrm{t}, 0.2,0.2)$ \\
& $\begin{array}{l}\text { ton of } \\
\mathrm{a}_{12}\end{array}$ & 4 & $\mathrm{~N} \sim(4,0.8)$ & $(\mathrm{t}, 0.2,0.2)$ \\
& $\begin{array}{l}\text { waste/tonne } \\
\mathrm{a}_{21}\end{array}$ & & & \\
$\mathrm{a}_{22}$ & hectare/tonne & 3 & $\mathrm{~N} \sim(3,0.5)$ & $(\mathrm{t}, 0.2,0.2)$ \\
$\mathrm{b}_{1}$ & hectare/tonne & 2 & $/$ & $(\mathrm{t}, 0.2,0.2)$ \\
$\mathrm{b}_{2}$ & tonne/week & 140 & $\mathrm{~N} \sim(140,10)$ & $(\mathrm{t}, 10,10)$ \\
\hline
\end{tabular}

\section{Solution Algorithm}

The key steps of the solution algorithm are as follows:

Step 1. Formulate the fuzzy model (Equation 1).

Step 2. Initialize the model parameters, including probability distributions and membership functions.

Step 3. Generate a set of random variables according to the probability distributions.

Step 4. Transform the Equation (1) to Equation (8) according to the generated random variables in Step 3.

Step 5. Solve Equation (8) and obtain the corresponding $X_{j l, o p t}$, and $f_{l, o p t}$ of the current trial.

Step 6 . Go to Step 7 if the trial reaches the preset number of trials $(l=N)$; otherwise $(l<N)$ go to Step 3 .

Step 7. Obtain a set of feasible solutions by Equation (9) or declare the feasible solutions are unachievable.

Step 8. Obtain the optimal solutions by Equation (12): $X_{j, o p t}=E\left(X_{j l, o p t}\right)$, and $f_{\text {opt }}=E\left(f_{l, o p t}\right)$.

Step 9. End

\section{A Case Study}

Consider a composting facility which generates two types of soil conditioners $\left(F_{1}\right.$ and $\left.F_{2}\right)$ based on two different types of composting technologies. When each tonne of $F_{1}$ and $F_{2}$ is generated, the required amount of MSW is $a_{11}$ and $a_{12}$ (tonne), respectively. It is assumed that the treated amount of waste per week should not be lower than the waste generation amount $\left(b_{1}\right.$, tonnes/week) of the city. It is estimated that one tonne of $\mathrm{F}_{1}$ can feed $a_{2 l}$ hectares of farms, and one tonne of $\mathrm{F}_{2}$ can feed $a_{22}$ hectares of farms. Due to the contract with the local farmers, the composting facility should supply sufficient soil conditioners to farmers to feed at least $b_{2}$ hectares of farms every week. The question is to determine the production rate ( $x_{1}$ and $x_{2}$, tonne/week) of two types of soil conditioners with the minimum system cost $\left(f, \$ 10^{3} /\right.$ week). Accordingly, an optimization model is formulated as follows:

Min $f=c_{1} x_{1}+c_{2} x_{2}$

Subject to:

$$
a_{11} x_{1}+a_{12} x_{2} \geq b_{1}
$$

$a_{21} x_{1}+a_{22} x_{2} \geq b_{2}$

$x_{1}, x_{2} \geq 0$

where $c_{1}$ and $c_{2}$ are the unit cost $\left(\$ 10^{3} /\right.$ tonne) for producing $\mathrm{F}_{1}$ and $\mathrm{F}_{2}$, respectively.

Due to subject judgement and incomplete information, possibilistic and continuously probabilistic uncertainties coexist in some of the model parameters. According to the historical information and literature the estimations of model parameters were obtained and shown in Table 1.

The unit costs $\left(c_{1}\right.$ and $\left.c_{2}\right)$ are deterministic values; possibility exists in all the left hand-side coefficients with a form of $(t, 0.2,0.2)$, indicating a value $t$ with the highest degree of likelihood, and the value of 0.2 for both the left and right spreads; possibility exists in all the RHS coefficients with a form of $(t$, $10,10)$, indicating a value $t$ with the highest degree of likelihood, and the value of 10 for both the left and right spreads; probability exists in $a_{12}, a_{21}, b_{1}$, and $b_{2}$ in the form of normal distributions, and they also contain possibilistic uncertainties, leading to dual uncertainties of possibility and continuous probability.

According to Equation (8) and the membership provided in Table 1, the original model (Equation 13) is converted to the following one:

Min $f=3 x_{1}+2 x_{2}-\lambda_{1}-\lambda_{2}$

Subject to:

$\frac{1}{2}\left[b_{1}-x_{1}-a_{12} x_{2}+0.2 x_{1}+0.2 x_{2}+10\right]=\lambda_{1}$

$\frac{1}{2}\left[b_{2}-a_{21} x_{1}-2 x_{2}+0.2 x_{1}+0.2 x_{2}+10\right]=\lambda_{2}$

$0 \leq \lambda_{1}, \lambda_{2} \leq \frac{1}{2}\left[0.2 x_{1}+0.2 x_{2}+10\right]$

$x_{1}, x_{2} \geq 0$

The preset number of trials is 1,000 . In each trial the random values for $a_{12}, a_{21}, b_{1}$, and $b_{2}$ are assigned by the randomization process and the optimal solution for each trial is obtained based on the steps of the solution algorithm described previously.

\section{Results and Discussion}

Feasible solutions were obtained in 997 out of 1,000 trails, indicating a high efficiency of the proposed model in solving the problem with the dual uncertainties of possibility and con- 


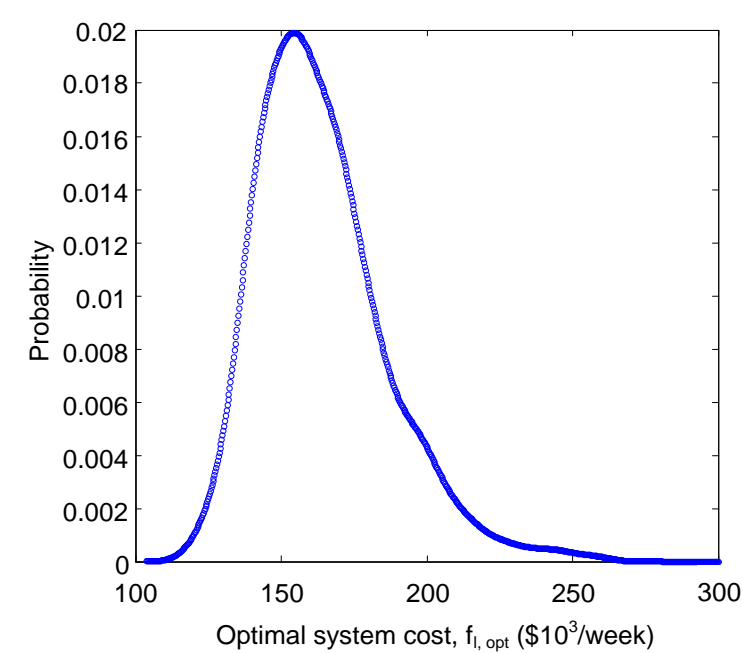

Figure 3. Distribution of the optimal system cost, $f_{l, \text { opt }}$.

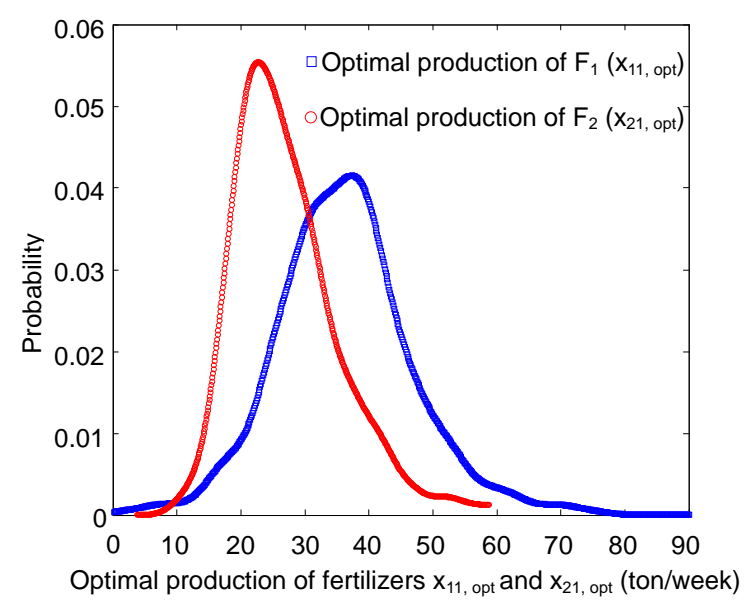

Figure 4. Distribution of the optimal production of the two soil conditioners $\left(x_{1 l, \text { opt }}\right.$ and $\left.x_{2 l, o p t}\right)$.

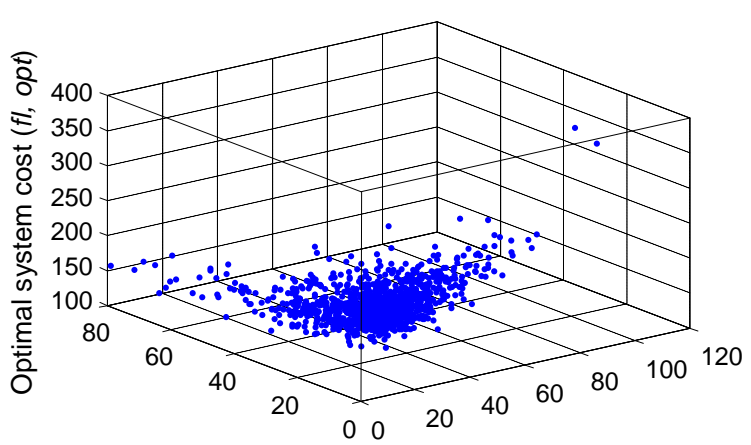

Production of $F_{2}$ ( $x_{21, \text { opt }}$, tonne/week) Production of $F_{1}\left(x_{21, \text { opt }}\right.$, tonne/week)

Figure 5. Relationship between optimal system cost and production of soil conditioners.

tinuous probability. The distributions of the results of the optimal system cost $\left(f_{l, o p t}\right)$ and the optimal production of soil conditioners $\left(x_{l, \text { opt }}\right)$ are shown in Figures 3 and 4. The figures indicate that all the distributions of the optimal solutions tend to be the lognormal distribution. This is significantly different from the original distributions of the inputs which are all normal distributions. The $95 \%$ confidence intervals indicate that the optimal production of $\mathrm{F}_{1}\left(x_{l l, o p t}\right)$ is mostly distributed between 15 and 60 tonne/week; the production of $\mathrm{F}_{2}\left(x_{2 l, \text { opt }}\right)$ is mostly distributed between 13 and 45 tonne/week; and the most frequently occurring optimal system cost $\left(f_{l, \text { opt }}\right)$ is in the range of 120 and 220 ( $\$ 10^{3} /$ week). Figure 5 indicates that most of the data points (optimal solutions) are within the same range and also shows the relationship between the optimal system cost and production of soil conditioners.

According to the Definition 1, the expected optimal minimum system cost would be $160.4\left(\$ 10^{3} /\right.$ week) under the dual uncertainties of possibility and continuous probability, and such a cost could be achieved based on the production rate of 35.8 tonnes $F_{1}$ and 25.8 tonnes of $F_{2}$ per week.

In order to test the sensitivity and robustness of the developed method with consideration of dual uncertainties, further analyses were conducted to calculate the expected values and standard deviations of the optimal system costs when the spreads of memberships and probability distributions were changed from -1.0 to 1.0 (or -100 to $100 \%$ with $10 \%$ interval), respectively (Figures 6 and 7). Furthermore, distri- butions of the optimal system costs were generated based on the changes of memberships and probability distributions from -1 to 1 with 0.4 interval (-100 to $100 \%$ with $40 \%$ interval) (Figure 8). The results indicated that the expected values of the optimal system cost were relatively sensitive to the possibilitic uncertainty but robust to the probabilistic uncertainty (Figure 6). Meanwhile, the disperstiveness represented by the standard deviations in this case of the optimal system cost were significantly sensitive to the probabilistic uncertainty but robust to the possibilistic uncertainty (Figure 8). In the other words, when the spread of the probability distribution increased, the expected value almost did not change and the disperstiveness significantly increased. In contrast, when the spread of membership increased, the expected value signifycantly decreased and the disperstiveness almost did not change. The similar conclusion could be supported by the distribution of optimal solutions according to changes of spreads of the probability distribution and membership function (Figure 8). The increase of the spread of the probability distribution could flat the shape of the optimal system cost distribution with unchanged expected value (Figure 8a). Comparatively, the increase of the membership spread could decrease the expected value without affecting the shape of distribution (Figure 8b).

By using the proposed MCFP method, when data are insufficient to accurately determine the probability distribution, the expected value of optimal system cost would not be intensively affected by subjectively selected probability distribution, and it just could affect the confidence interval of the expected value. Consequently, although the insufficient data could not accurately be determined to certain probability distribution, the proposed methods could still provide unbiased expected optimal system cost. On the other hand, different experts/managers could provide different suggestions on possibility/membership functions, and it would affect the expected value of the optimal 


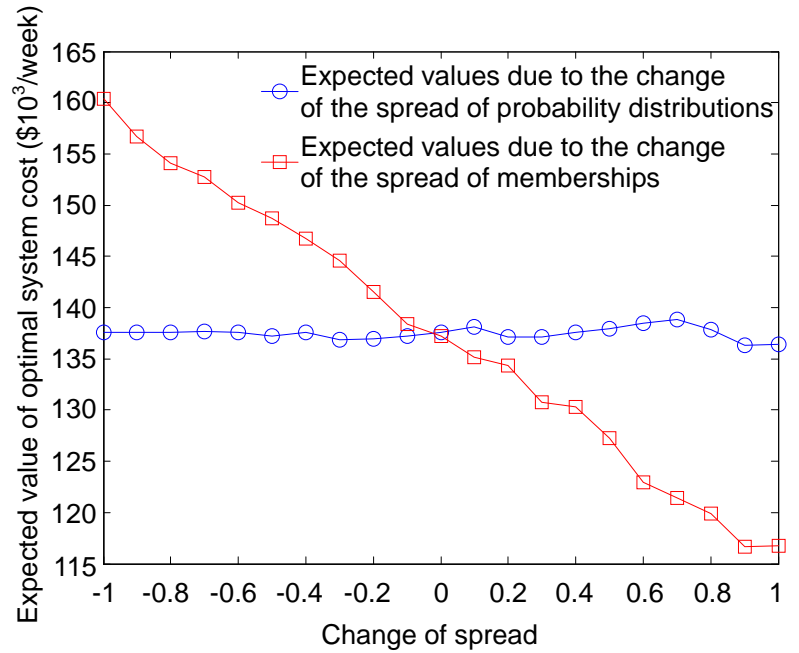

Figure 6. The expected values of the optimal system cost with spreads of probability distribution and memberships changing from $-100 \%$ to $100 \%$ based on 1,000 trials of Monte Carlo simulation.

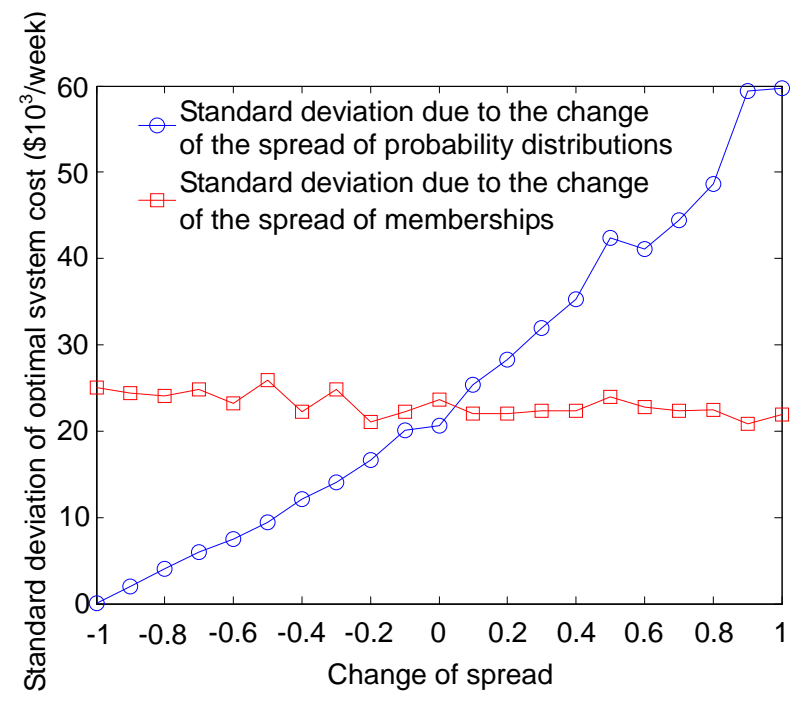

Figure 7. The standard deviations of the optimal system cost with spreads of probability distribution and memberships changing from $-100 \%$ to $100 \%$ based on 1,000 trials of Monte Carlo simulation.

system cost but would not dramatically influence probability and confidence interval of expected values. In the other words, subjective information collected from experts/managers is likely to cause variations in optimal system cost without affecting its confidence intervals. Therefore, even though the subjective information from experts/managers are very crucial to expected optimal system cost, the proposed optimization methods could still robustly provide fairly constant confidence interval (range of optimal system cost) for the expected cost.

It is worth note that the solutions can provide three types of decision supports to help different levels of decision makers regulate, manage and/or design, and operate the MSW management system. Firstly, entire distribution of the optimal sys-
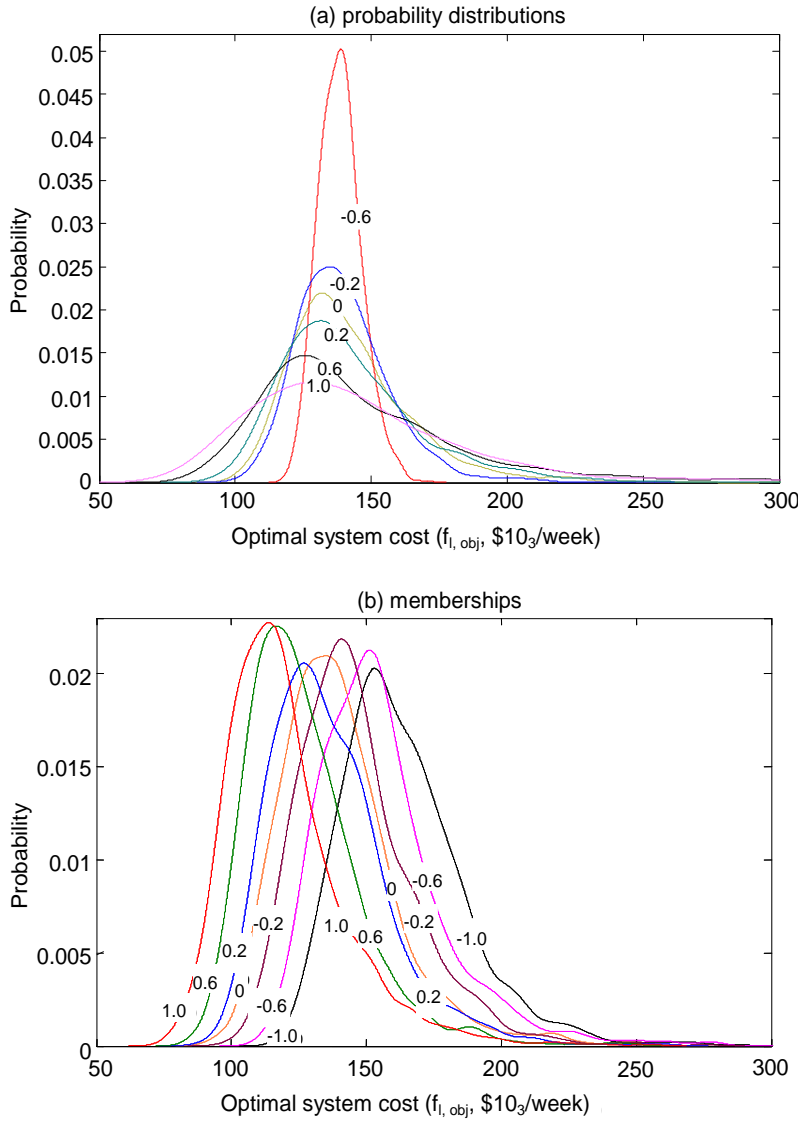

Figure 8. Distributions of the optimal results with the spreads of (a) probability distributions and (b) memberships changing from $-100 \%$ to $100 \%$ based on 1,000 trials of Monte Carlo simulation.

tem (Figure 3) cost and corresponding soil conditioners production (Figure 4) can provide a complete image to local authorities for legislating/adjusting relative policies and regulations and developing strategies for MSW management; Secondly, the range of most frequent occurrences (15 and 60 tonne/week of $F_{1}$ and 13 and 45 tonne/week of $F_{2}$ could lead to the optimal system cost of $[120,220] * \$ 10^{3} /$ week) can assist the facility managers to flexibly and effectively develop the production plan. Thirdly, the expected values of the optimal system cost and the corresponding production (production rate of 35.8 tonnes $F_{1}$ and 25.8 tonnes of $F_{2}$ lead to optimal system cost of $160.4 * \$ 10^{3}$ for per week) can directly and clearly provide sound decisions to the facility.

Furthermore, as two important measures in engineering optimization, accuracy and efficiency usually conflict and require some compromises with each other. Currently there is no clear rule for the setting of the number of trials for Monte Carlo simulation. Nevertheless, according to Driels and Shin (2004), when the number of trials is higher than 300, Monte Carlo simulation can provide reasonable results. In addition, Winston (2000) also claimed that a minimum number of 450 trials would be required for the Monte Carlo simulation to achieve $95 \%$ of accuracy of the estimation, and the results could 
be reliable when the number of trials is up to 1,000 . However, with the increasing number of trials, the requirement of computational resource and time consumption significantly increases. In order to achieve a reliably accurate result with minimum trials, the case study was further conducted based on different trials of Monte Carlo simulation (100 to 1,000 with an interval of 100 trials; 1,000 to 10,000 with an interval of 1000 trials). The results indicated significant differences in system cost from 100 to 1,000 trials and insignificant differences when the trial numbers were higher than 1,000 . The difference of system costs from 1,000 and 10,000 trials optimization was less than $0.1 \%$, however, the time consumption was more than 20 times from 1,000 trials (20 min) to 10,000 trials (450 min). Therefore, 1,000 trials of Monte Carlo simulation will be the optimal option for the case study.

\section{Conclusions}

Decision making in environmental management can be complex (Huang and Loucks, 2000; Huang et al., 2006; Qin et al., 2007; Li et al., 2008a,b; Li et al., 2010; Lv et al., 2010). This study developed a Monte Carlo simulation-based fu- zzy programming (MCFP) to reflect and quantify the dual uncertainties of possibility and continuous probability in environmental management. The fuzzy programming approach was advanced from the fuzzy-stochastic-interval linear programmming (FSILP) approach which was previously developed by the authors. The approach is highly capable of converting fuzzy problems to deterministic ones and achieving optimal solutions with fewer additional constraints, leading to significant reduction of computation time. Another important novelty of this approach is the integration of fuzzy programming and Monte Carlo simulation, which can effectively tackle the coexistence of uncertainties in forms of fuzzy sets and continuous probability distributions. The developed approach was further tested by a case study of MSW management. The results demonstrated that the MCFP approach could effectively integrate fuzzy programming and Monte Carlo simulation to deal with subjective judgement from experts and incomplete information represented by fuzzy membership and continuous probability distributions. Therefore, the MCFP approach is capable of providing decision support to management problems that involve coexistence of dual uncertainties.

In addition, the developed approach can provide three levels of the optimal results to help the decision maker effectively manage the system. The first level is the entire distributions of objective functions and decision variables, which can provide decision supports to general policy makers (e.g., regulating and consulting organizations) for long term policy making and trade-off, risk and reliability analyses of the system; the second level is the range of most frequent occurrences, which can help project or plant managers in designing and planning the production in a medium arrangement; the third level is the expected value of the optimal results, which can directly provide decision alternatives to the plant operators for short term operating and adjusting the facility to minimize system cost.
Future research may focus on how to handle additional uncertainties existing in the coefficients of the objective function and how to integrate other forms of uncertainties (e.g., intervals) into decision making processes to deal with more uncertain conditions. The approach will be further tested through real-world cases.

\section{Nomenclature}

$\delta$ - lower spread of membership function

$\delta^{+} \quad$ upper spread of membership function

$\lambda \quad$ matrix of control decision variables

$\sim \quad$ indicator for possibilistic uncertainty

A matrix of constraint coefficients

a amount of MSW for composting, tonne/tonne

$A^{l} \quad$ matrix of positive constraint coefficients

$A^{2}$ matrix of negative constraint coefficients

$B$ resources of constraints

$b$ waste generation amount, tonne/week

$B^{l}$ matrix of positive RHSs

$B^{2}$ matrix of negative RHSs

$C$ coefficients of the objective function

$c$ cost of soil conditioner production, $\$ 10^{3} /$ tonne

$E$ function of mathematical expectation

$f$ objection function (system cost in the case study, $\$ 10^{3} /$ week)

F soil conditioner

$l \quad$ index of the current trial

LHS left-hand-side

$M \quad$ number of the feasible solutions

$m$ number of constraints

MSW municipal solid waste

$N$ preset number of trials

$n \quad$ number of decision variables

$p \quad$ matrix of probability

RHS right-hand-side

$S \quad$ matrix of slick variables

$t$ most likely value of membership function

$w \quad$ indicator for probabilistic uncertainty

$X$ matrix of decision variables

$x \quad$ soil conditioner production rate, tonne/week

$Z \quad$ number of decision variables

Acknowledgments. The research work was supported by the National Key Research and Development Plan (2016YFA0601502, 2016YFC0502800), the National Natural Science Foundation of China (No. 51179070) and Natural Science and Engineering Research Council of Canada. The authors deeply appreciate the editors and the anonymous reviewers for their insightful comments and suggestions. 


\section{References}

Anderson, H.L. (1986). Metropolis, Monte Carlo and the MANIAC. Los Alamos Sci., 14, 96-108.

Baeurle, S.A. (2009). Multiscale modeling of polymer materials using field-theoretic methodologies: A survey about recent developments. J. Math. Chem., 46(2), 363-426. http://dx.doi.org/10.100 7/s10 910-008-9467-3

Baudrit, C., Guyonnet, D., and Dubois, D. (2007). Joint propagation of variability and imprecision in assessing the risk of groundwater contamination. J. Contam. Hydrol., 93, 72-84. http://dx.doi.org/10. 1016/j.jconhyd.2007.01.015

Blair, A.N., Ayyub, B.M., and Bender, W.J. (2001). Fuzzy stochastic risk-based decision analysis with the mobile offshore base as a case study. Mar. Struct., 14(1), 69-88. http://dx.doi.org/10.1016/S095 1-8339(00)00027-7

Chen, B., Guo, H.C., and Huang, G.H. (2008). IFMEP: An interval fuzzy multiobjective environmental planning model for watershed systems. Civ. Eng. Environ. Syst., 25(2), 99-125. http://dx.doi.org/ $10.1080 / 10286600801908899$

Chen, Z., Huang, G.H., and Chakma, A. (2003). Hybrid fuzzy stochastic modeling approach for assessing environmental risks at contaminated groundwater system. J. Environ. Eng., 129, 79. http://dx.doi.org/10.1061/(ASCE)0733-9372(2003)129:1(79)

Cheng, G.H., Huang, G.H., Cao, M.F., and Fan, Y.R. (2009). Planning of municipal solid waste management systems under dual uncertainties: A hybrid interval stochastic programming approach. Stochastic Environ. Res. Risk Assess., 236, 702-720. http://dx.doi.org/ 10.1007/s00477-008-0251-5

Driels, M.R. and Shin, Y.S. (2004). Determining the Number of Iterations for Monte Carlo Simulations of Weapon Effectiveness, Naval Postgraduate School, United States, pp. 16.

Freeze, A., Massmann, J., Smith, L., Sperling, T., and James, B. (1991). Hydrogeological decision analysis: 1. A framework. Ground Water, 28 (5), 738-766. http://dx.doi.org/10.1111/j.1745- 6584. 1990.tb01989.x

Garthwaite, P., Kadane, J., and O'Hagan, A. (2005). Statistical methods for eliciting probability distributions. J. Am. Med. Assoc., 100, 680-701. http://dx.doi.org/10.1198/016214505000000105

Goldstein, M. (2006). Subjective Bayesian analysis: Principles and practice. Int. Soc. Bayesian Anal., 1(3), 403-420.

Guo, P. and Huang, G.H., (2009). Two-Stage Fuzzy Chance-Constrained Programming: Application to Water Resources Management under Dual Uncertainties. Stochastic Environ. Res. Risk Assess., 23:349-359. http://dx.doi.org/10.1007/s00477-008-0221-y

Guyonnet, D., Bourgigne, B., Dubois, D., Fargier, H., Come, B., and Chiles, J. (2003). Hybrid approach for addressing uncertainty in risk assessments. J. Environ. Eng., 129(1), 68-78. http://dx.doi.org/ 10.1061/(ASCE)0733-9372(2003)129:1(68)

Huang, G.H. (1998). A hybrid inexact-stochastic water management model. Eur. J. Oper. Res., 107(1), 137-158. https://doi.org/10.1016/ S0377-2217(97)00144-6

Huang, G.H., Huang, Y.F., Wang, G.Q., and Xiao, H.N. (2006). Development of a forecasting system for supporting remediation design and process control based on NAPL-biodegradation simulation and stepwise-cluster analysis. Water Resour. Res., 42(6). https://doi.org/10.1029/2005WR004006

Huang, G.H., and Loucks, D.P. (2000). An inexact two-stage stochastic programming model for water resources management under uncertainty. Civ. Eng. Environ. Syst., 17(2), 95-118. https:// doi.org/10.1080/02630250008970277

Huang, G.H., Sae-Lim, N., Liu, L., and Chen, Z. (2001). An intervalparameter fuzzy-stochastic programming approach for municipal solid waste management and planning. Environ. Model. Assess., 6,
271-283. http://dx.doi.org/10.1023/A:1013394118863

Jing, L., Chen, B., Zhang, B.Y., Li, P., and Zheng, J.S. (2013). Monte Carlo simulation-aided analytic hierarchy process approach: Case study of assessing preferred non-point-source pollution control best management practices. ASCE J. Environ. Eng., 139(5), 618-626. http://dx.doi.org/10.1061/(ASCE)EE.1943-7870.0000673

Karmperis, A.C., Sotirchos, A., Aravossis, K., and Tatsiopoulos, I.P. (2012). Waste management project's alternatives: A risk-based multi-criteria assessment (RBMCA) approach. Waste Manage., 32(1), 194-212. http://dx.doi.org/10.1016/j.wasman.2011.09.001

Li, J.B., Huang, G.H., Zeng, G.M., and Maqsood, I. (2004). Stochastic risk assessment of groundwater contamination: A western Canadian case study. Environ. Inf. Arch., 2 (2004), 1021-1032.

Li, J.B., Huang, G.H., and Zeng, G., (2006). A fuzzy-set approach for addressing uncertainties in risk assessment of hydrocarbon-contaminated site. Water Air Soil Pollut., 171(1-4), 5-18. http://dx.doi. org/10.1007/s11270-005-9005-x

Li, J.B., Huang, G.H., Zeng, G., Maqsoodd, I., and Huang, Y.F. (2007). An integrated fuzzy-stochastic modeling approach for risk assessment of groundwater contamination, J. Environ. Manage., 82, 173188. http://dx.doi.org/10.1016/j.jenvman.2005.12.018

Li, P., and Chen, B. (2011). FSILP: Fuzzy-stochastic-interval linear programming for supporting municipal solid waste management. $J$. Environ. Manage., 92, 1198-1209. http://dx.doi.org/10.1016/j.jenv man. 2010.12.013

Li, P., Chen, B., Zhang, B.Y., Jing, L., and Zheng, J.S. (2014). Monte Carlo simulation-based dynamic mixed integer nonlinear programming for supporting oil recovery and devices allocation during offshore oil spill responses. Ocean Coast. Manage., 89C (2014), 5870. http://dx.doi.org/10.1016/j.ocecoaman.2013.12.006

Li, T., Li, P., Chen, B., Hu, M., and Zhang, X. (2012). A simulationbased inexact two-stage chance constraint quadratic programming for sustainable water quality management under dual uncertainties. ASCE J. Water Resour. Plann. Manage., 140(3), 298-312. http://dx. doi.org/10.1061/(ASCE)WR.1943-5452.0000328

Li, Y.P., Huang, G.H. (2009). Fuzzy-stochastic-based violation analysis method for planning water resources management systems with uncertain information. Inform. Sciences, 179, 4261-4276. http://dx. doi.org/. 10.1016/j.ins.2009.09.001

Li, Y.P., Huang, G.H., Huang, Y.F., and Zhou, H.D. (2009). A multistage fuzzy-stochastic programming model for supporting sustainable water-resources allocation and management. Environ. Model. Software, 24, 786-797. http://dx.doi.org/10.1016/j.envsoft.2008.11. 008

Li, Y.P., Huang, G.H., and Nie, S.L. (2010). Planning water resources management systems using a fuzzy-boundary interval-stochastic programming method. Adv. Water Resour., 33(9), 1105-1117. https://doi.org/10.1016/j.advwatres.2010.06.015

Li, Y.P., Huang, G.H., Nie, S.L., and Liu, L. (2008a). Inexact multistage stochastic integer programming for water resources management under uncertainty. J. Environ. Manage., 88(1). 93107. https://doi.org/10.1016/j.jenvman.2007.01.056

Li, Y.P., Huang, G.H., and Sun, W. (2011). Management of uncertain information for environmental systems using a multistage fuzzystochastic programming model with soft constraints. J. Environ. Inf., 18(1), 28-37. http://dx.doi.org/10.3808/jei.201100196

Li, Y.P., Huang, G.H., Yang, Z.F., and Nie, S.L. (2008b). IFMP: Interval-fuzzy multistage programming for water resources management under uncertainty. Resour. Conserv. Recycling, 52(5), 800-812. https://doi.org/10.1016/j.resconrec.2007.11.007

Lin, G.H., Chen, X.J., and Fukushima, M. (2009). Solving stochastic mathematical programs with equilibrium constraints via approximation and smoothing implicit programming with penalization. Math. Programming, 116, 343-368. http://dx.doi.org/10.1007/s101 
07-007-0119-3

Liu, L., Cheng, S.Y., and Guo, H.C. (2004). A simulation-assessment modeling approach for analyzing environmental risks of groundwater contamination at waste landfill sites. Hum. Ecol. Risk Assess., 10(2), 373-388. http://dx.doi.org/10.1080/10807030490438436

Liu, Z.F., Huang, G.H., Nie, X.H., and He, L. (2009). Dual-interval linear programming model and its application to solid waste management planning. Environ. Eng. Sci., 26(6), 1033-1045. http://dx. doi.org/10.1089/ees.2007.0289

Lv, Y., Huang, G.H., Li, Y.P., Yang, Z.F., Liu, Y., and Cheng, G.H. (2010). Planning regional water resources system using an interval fuzzy bi-level programming method. J. Environ. Inf., 16(2), 43-56. https://doi.org/10.3808/jei.201000177

Ping, J., Chen, Y., Chen, B., and Howboldt, K. (2010a). A robust statistical analysis approach for pollutant loadings in urban rivers. J. Environ. Inf., 16(1), 35-42. http://dx.doi.org/10.3808/jei.20100 0176

Ping, J., Chen, B., and Husain, T. (2010b). Risk assessment of ambient air quality by stochastic-based fuzzy approaches. Environ. Eng. Sci., 27(3), 233-246. http://dx.doi.org/10.1089/ees.2009.0350

Qin, X.S., and Huang, G.H. (2008). Characterizing uncertainties associated with contaminant transport modeling through a coupled fuzzy-stochastic approach. Water Air Soil Pollut., 197(1-4), 331-348.

Qin, X.S., Huang, G.H., Zeng, G.M., Chakma, A., and Huang, Y.F. (2007) An interval-parameter fuzzy nonlinear optimization model for stream water quality management under uncertainty. Eur. $J$. Oper. Res., 180(3), 1331-1357. https://doi.org/10.1016/j.ejor.2006. 03.053

Ramik, J., and Vlach, M. (2004). Fuzzy mathematical programming: a unified approach based on fuzzy relations. Fuzzy Optimiz. Decis. Making, 1(4), 335-346. http://dx.doi.org/10.1023/A:1020978428453

Sadeghi, N., Fayek, A.R., and Pedrycz, W. (2010). Fuzzy Monte Carlo simulation and risk assessment in construction. Comput. Aided Civ. Infrastruct. Eng., 25, 238-252. http://dx.doi.org/10.11
$11 / j .1467-8667.2009 .00632 . x$

Seuntjens, P. (2002). Field-scale cadmium transport in a heterogeneous layered soil. Water Air Soil Pollut., 140(1-4), 401-423 http:// dx.doi.org/10.1023/A:1020147610743

Tan, Z.F., Song, Y.H., Shen, Y.S., Zhang, C., and Wang S. (2013). An optimization-based study to analyze the impacts of clean energy and carbon emission mechanisms on inter-regional energy exchange. J. Environ. Inf., 22(2), 123-130. http://dx.doi.org/10.3808/ jei.201300251

Van Hop, N. (2007a). Fuzzy stochastic goal programming problems. Eur. J. Oper. Res., 176: 77-86. http://dx.doi.org/10.1016/j.ejor.2005. 09.023

Van Hop, N. (2007b). Solving fuzzy (stochastic) linear programming problems using superiority and inferiority measures. Inf. Sci., 177: 1977-1991. http://dx.doi.org/10.1016/j.ins.2006.12.001

Van Hop, N. (2007c). Solving linear programming problems under fuzziness and randomness environment using attainment values. Inf. Sci., 177, 2971-2984. http://dx.doi.org/10.1016/j.ins. 2007.01. 032

Vose, D. (1996). Quantitative Risk Analysis: A Guide to Monte Carlo Simulation Modelling, John Wiley \& Sons, New York.

Winston, W.L. (2000). Simulation Modeling Using @RISK, Cengage Learning, pp. 226.

Xu, Y., Huang, G.H., Qin, X.S., and Cao, M.F. (2009). SRCCP: A stochastic robust chance-constrained programming model for municipal solid waste management under uncertainty. Resour. Conserv. Recycling, 53, 352-363. http://dx.doi.org/10.1016/j.rescon rec. 2009.02.002

Yang, A.L., Huang, G.H., and Qin, X.S. (2010). An integrated simulation-assessment approach for evaluating health risks of groundwater contamination under multiple uncertainties. Water Resour. Manage., 24(13), 3349-3369. http://dx.doi.org/10.1007/s11269-01 0-9610-3 